\title{
Interactive Procedure for Localized Monitoring and Control of Polyline Simplification
}

\author{
Barry Kronenfeld ${ }^{\mathrm{a}, *}$, Lawrence Stanislawski ${ }^{\mathrm{b}}$, Barbara P. Buttenfield ${ }^{\mathrm{c}}$, Ethan Shavers ${ }^{\mathrm{b}}$ \\ ${ }^{a}$ Eastern Illinois University, Charleston Illinois, United States, bjkronenfeld@eiu.edu \\ ${ }^{b}$ U.S. Geological Survey, Center of Excellence for Geospatial Information Science, Rolla Missouri, United States, \\ lstan@usgs.gov,Ethan Shavers, eshavers@usgs.gov \\ ${ }^{c}$ University of Colorado,Boulder Colorado,United States, babs@colorado.edu \\ * Corresponding author
}

Keywords: vector generalization, simplification, smoothing, area displacement, Hausdorff distance

\begin{abstract}
:
This research develops and tests procedures for interactive simplification of polyline features with near real-time assessment capabilities. The process enables a user to manually combine various simplification operations until a suitable solution is found that is tailored for their particular use, such as a topographic map, a thematic representation, or a schematic diagram. Available operations include dynamic parameterization of automated algorithms, spot tools for manual local feature modification, and real-time feedback on assessment metrics. All operations are designed within an area-preserving framework to limit deviation from the original polylines. As a pilot study, these procedures were used to simplify a small sample of vector stream data from the United States National Hydrography Dataset (NHD) for display on thematic and reference maps. These data are provided as a benchmark for other researchers (DOI: 10.5281/zenodo.5818077).
\end{abstract}

The test dataset for this demonstration was extracted from NHD features within the boundary of the 1:24,000-scale (24k) Sheep Creek Dam topographic quadrangle in North Dakota (Figure 1), which includes part of the Cannonball River. The NHD is a comprehensive vector dataset representing surface water features in the United States that are compiled from $24 \mathrm{k}$ or larger scale source data $(1: 63,360$-scale or larger in Alaska). NHD features are assigned a VisibilityFilter value in feature attribute tables that enables automated selection of NHD features to generalize data to a series of smaller scales, while retaining natural density variations (Stanislawski and others, 2012; Stauffer and others, 2016). The original 24k data contained 227 flowline features, including 133 stream/river features and 94 artificial path features that connect the flow network. The 227 features were reduced to 28 and 7 features for display at 1:100,000 $(100 \mathrm{k})$ and 1:500,000 (500k) scales, respectively, through a series of filtering and consolidation steps (Table 1).

\begin{tabular}{|l|c|c|}
\hline \multirow{2}{*}{ Processing Step } & \multicolumn{2}{|c|}{ remaining features } \\
\cline { 2 - 3 } & $\mathbf{1 0 0 k}$ & $\mathbf{5 0 0 k}$ \\
\hline original 24k data & 227 & 227 \\
\hline visibility field selection & 137 & 55 \\
\hline extraction of contiguous network & 90 & 52 \\
\hline consolidation of consecutive reaches & 28 & 7 \\
\hline
\end{tabular}

Table 1. Processing steps to filter and consolidate $24 \mathrm{k}$ flowline features for $100 \mathrm{k}$ and $500 \mathrm{k}$ benchmark simplification.

Flowline features were first simplified using the area-preserving segment collapse (APSC) algorithm (Kronenfeld and others, 2020). Reduction was initialized to a target error (stepwise areal displacement) tolerance, but this was subject to manual adjustment by progressively simplifying the polylines as much as possible without removing visually noticeable meanders and bends at the target scale. Real-time feedback reported error values and vertex counts. Smoothing was applied by manually selecting vertices with high angularity and applying automated area-preserving smoothing techniques to each selected vertex (Tutić and LaPaine 2010). Three smoothing operations were available. Two involved vertex insertion and one vertex displacement. Smoothing was performed until the resulting polyline 
contained no noticeably sharp angles when displayed at $2 \mathrm{x}$ the target scale, and typically (but not always) resulted in a reduction in local displacement error. Third, locations approaching visual coalescence at $2 \mathrm{x}$ the target scale were identified visually, including stream confluences and narrow openings to large meanders. Exaggeration (separation) was performed at these locations to ensure legibility using the above area-preserving smoothing operations.

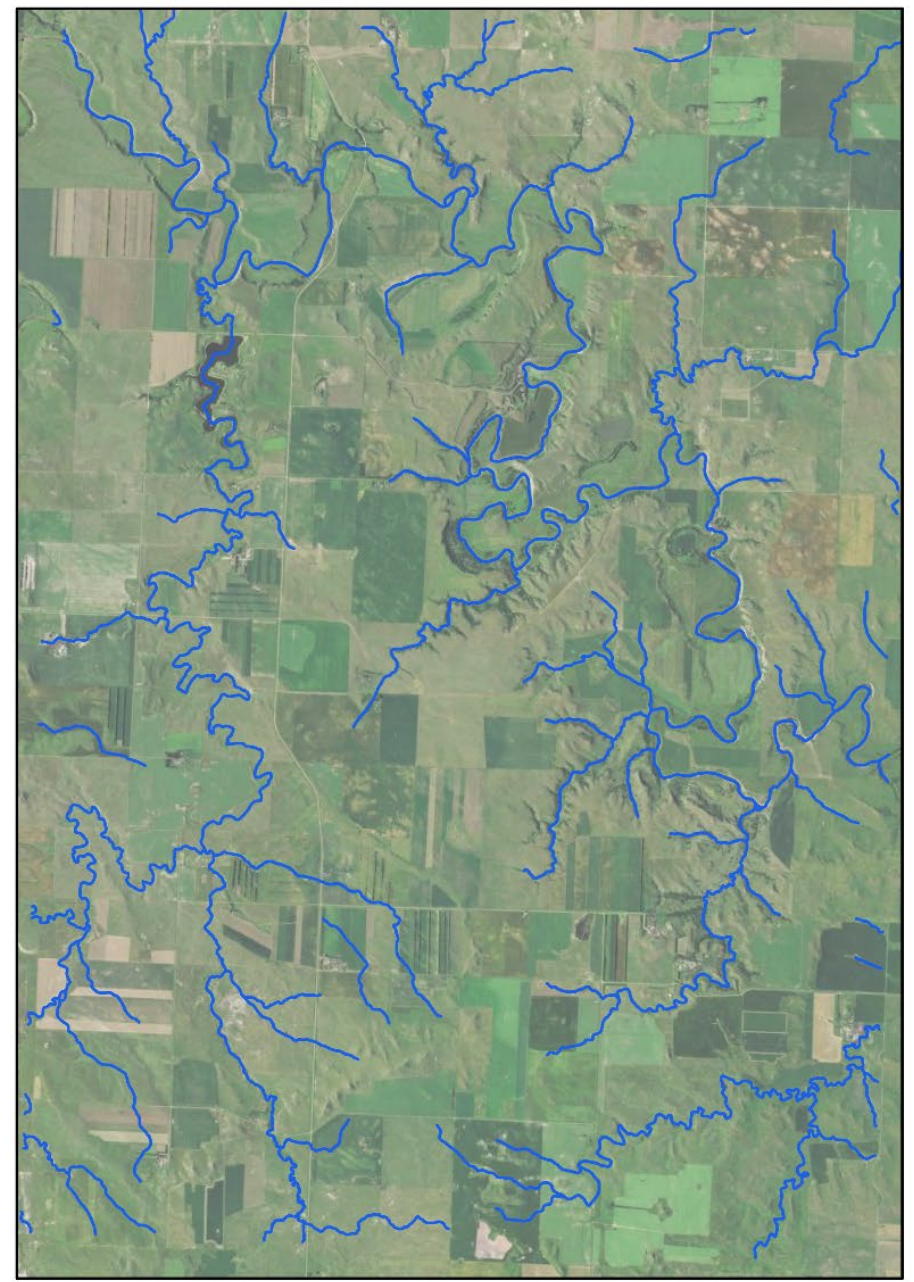

(a)

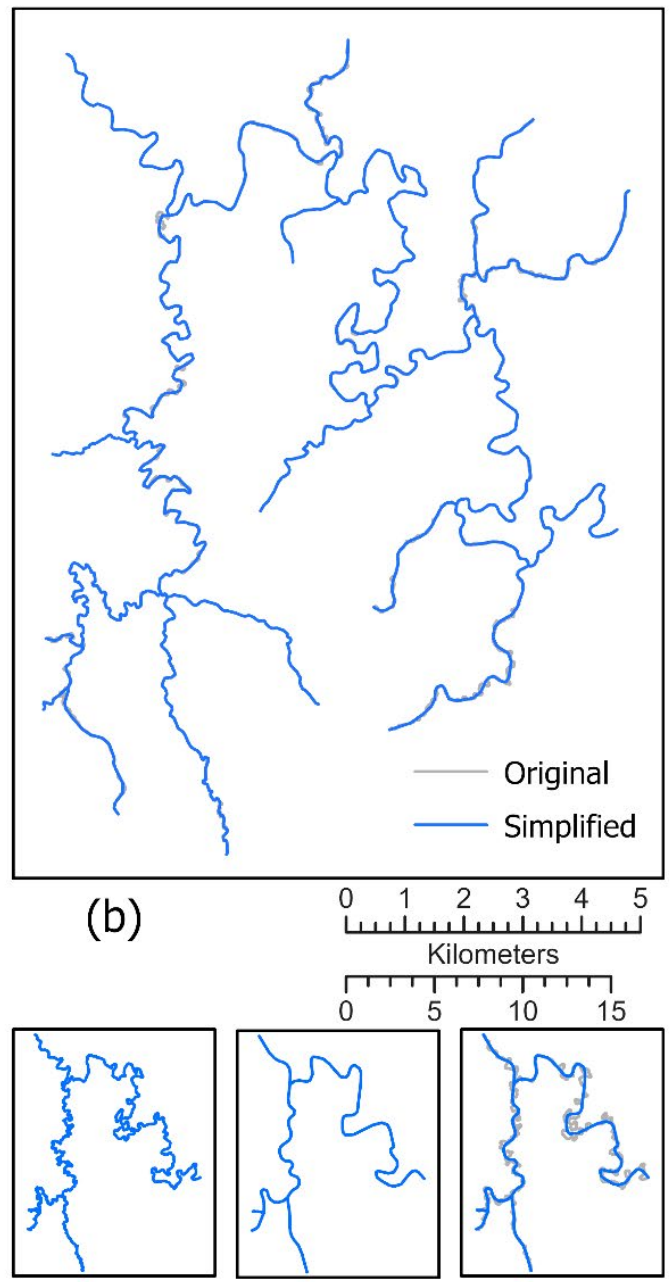

(c)

(d)

(e)

The original and benchmark simplified data are shown in Figure 1. The simplified data are smooth, legible and maintain high fidelity with respect to the original features. The simplified features appear suitable for use in reference and thematic maps. Several metrics to estimate generalization quality are being calculated. Specifically, we calculate both maximum and average distance between original and simplified polylines using the modified Hausdorff distance (Stanislawski et al. 2018) and the Vertex Influence Method (VIM) (Mozas and Ariza 2011). Departing from previous work, we separate the two directed components (i.e. the directed Hausdorff and the directed VIM) rather than taking the maximum of the two bidirectional components of these metrics. These may be treated as akin to producer's and 
consumer's (user's) accuracy measures (Aronoff 1982). Thus, we measure four different metrics of distance displacement: producer's modified Hausdorff distance, consumer's modified Hausdorff distance, producer's VIM and consumer's VIM. In addition, we measure changes in overall sinuosity and average angular deviation. These metrics may be used for evaluation of automated simplification procedures, which should strive to achieve similarly low values as the benchmark data for all four metrics. In addition, the number of retained vertices should be minimized to reduce computational requirements for data utilization.

The benchmark simplified data produced here form part of a project to develop a semi-automated simplification environment for producing high-quality generalizations by combining efficient computing procedures with human control and judgement. The environment will be shared at the workshop and several improvements are envisioned. First, additional real-time feedback metrics will be incorporated, including the metrics described above. Second, tools will be implemented to further automate smoothing. Third, additional tools will be created to facilitate feature separation/exaggeration. Suitable procedures to integrate changes across multiple data layers, including hydrography polygon features, are being researched.

\section{References}

Aronoff, S., 1982, Classification accuracy: a user approach. Photogrammetric Engineering and Remote Sensing, 48(8):1299-1307.

Kronenfeld, B.J., Stanislawski, L.V., Buttenfield, B.P. and Brockmeyer, T., 2020, Simplification of polylines by segment collapse: minimizing areal displacement while preserving area. International Journal of Cartography, 6(1):22-46

Kronenfeld, B.J., Stanislawski, L.V., Buttenfield, B.P. and Shavers, E., 2021, Sheep Creek Flowlines Generalized for 1:100,000 scale Representation (v1.0) [Data set]. Zenodo. https://doi.org/10.5281/zenodo.5818077

Mozas, A.T. and Ariza, F.J., 2011, New method for positional quality control in cartography based on lines. A comparative study of methodologies. International Journal of Geographical Information Science, 25(10):1681:1695.

Stanislawski, L.V., Doumbouya, A.T., Miller-Corbett, C.D., Buttenfield, B.P. and Arundel, S.T., 2012, Scaling stream densities for hydrologic generalization. Proceedings, 7th International Conference on Geographic Information Science, September 18-21, 2012, Columbus, Ohio, 6 p.

Stanislawski, L.V., Kronenfeld, B.J., Buttenfield, B.P. and Brockmeyer, T., 2018, Generalizing linear stream features to preserve sinuosity for analysis and display: a pilot study in multi-scale data science. In Freundschuh and Sinton (eds), Conference Proceedings, AutoCarto/UCGIS 2018. The $22^{\text {nd }}$ International Research Symposium on Computerbased Cartography and GIScience, Madison, WI, USA, May 22-24.

Stauffer, A.J., Finelli, E., and Stanislawski, L.V., 2016, Moving from generalization to the 'Visibility Filter Attribute': Leveraging database attribution to support efficient generalization decisions. American Water Resources Association 2016 Summer Specialty Conference, GIS \& Water Resources IX, July 11-13, 2016, Sacramento, California.

Tutić, D. and Lapaine, M., 2010, A new method for reducing sharp corners in cartographic lines with area preservation property, in: Naomi A, K Takashi, M Jun, S Aya and Y Yasushi (eds), Proceedings, 14th International Conference on Geometry and Graphics, Kyoto, 289-290. 